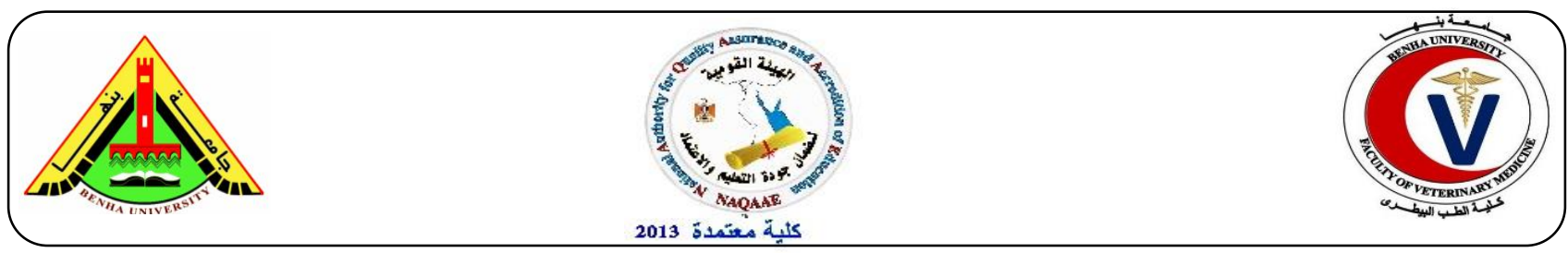

\title{
Field Evaluation of the prepared combined equine influenza, equine herpesvirus-1 and tetanus toxoid vaccine in horses
}

\author{
Mohamed Bayoumi ${ }^{1}$, Abdelfattah Selim ${ }^{2}$, Abdelmoneim M. Moustafa ${ }^{2}$, Nashwa K. Madkour ${ }^{1}$,El-Helw \\ H. A. ${ }^{3}$ \\ ${ }^{1}$ Equine Viral Disease Research Department, Veterinary Serum and Vaccine Research Institute, Abbasia \\ ${ }^{2}$ Animal Medicine Department, Faculty of Veterinary Medicine, Benha University \\ ${ }^{3}$ Anaerobic Bacterial Vaccine Department, Veterinary Serum and Vaccine Research Institute, Abbasia
}

\section{A B S T R A C T}

The use of vaccines in the prevention and control of infectious diseases in the horse is a widely accepted practice. A combined inactivated EIV, EHV-1 and tetanus toxoid vaccine adjuvanted with alhydrogel and saponin was evaluated in horses. Two groups of horses, group A was inoculated with two doses of the combined vaccine one month apart and revaccinated at 6 months and group B was kept as control. The immune response of vaccinated horses against EI, EHV-1 and tetanus was evaluated by measuring Haemagglutination Inhibition (HI), Enzyme linked immunosorbent assay (ELISA), Complement Fixation (CF) and toxin neutralizing antibody titers (TN). Vaccinated horses had high antibody titers, above the level for clinical protection against influenza and tetanus until 12 months and 6 months for EHV-1 after the third vaccination.

A vaccination scheme consisting of three doses with combined inactivated EI, EHV-1 and TT vaccine adjuvanted with saponin and alhydrogel, two doses at one-month interval and Revaccination after 6 months, Followed by alternating vaccinations with monovalent inactivated EHV-1vaccine and combined inactivated EI- EHV\& TT vaccine every 6 months is proposed to maintain maximal protection against equine influenza and herpesvirus and tetanus infections.

Keywords: EIV, EHV-1, tetanus toxoid, Horses, vaccine, immunity.

\section{INTRODUCTION}

Equine influenza virus (EIV) is a major respiratory pathogen of horses, causing high morbidity and occasional mortality. EI is a highly contagious disease contracted by inhalation of infectious virus aerosols. EI is not only an important welfare issue but can have a profound economic impact on the equine industry with major epidemics disrupting horse racing and breeding (Bryant et al., 2010).

Equine herpesvirus type $1(\mathrm{EHV}-1)$ is an alpha herpesvirus that causes a broad range of manifestations in horses, including a central nervous system disease involving the spinal cord and brain (myeloencephalopathy), respiratory disease, abortions, and perinatal death (Mesquita et al., 2017). 
Tetanus is a highly fatal, infectious disease of all species of domestic animals caused by the toxin of Clostridium tetani. The neurotoxin of C. tetani is exceedingly potent, but there is considerable variation in susceptibility between the animal species, the horse being the most susceptible (Jansen and Knoetze, 1979).

Toxin inactivated by formaldehyde (tetanus toxoid) is devoid of toxicity but is still highly immunogenic with a stabilized native conformation (Inić-Kanada et al., 2009).

The vaccine is important tool to control and prevent the most important distributed pathogens among equine. For many horses involved in competitive sport vaccination is mandatory and requires veterinary certification (Lunn and Townsend, 2000).

In addition to the selection of an appropriate virus strain, there are three important factors determining response to inactivated vaccines such as antigen content of the vaccine, adjuvant and vaccination regime (Holmes et al 2006).

The protection induced by first generations of whole inactivated, aluminum hydroxide adjuvanted EI vaccine primarily relied on stimulation of high antibody levels. Aluminum hydroxide is known to drive a $\mathrm{Th} 2$, antibody orientated immune response (Lindblad, 2004).

Saponin based adjuvants have the ability to modulate the cell mediated immune system as well as to enhance antibody production and have the advantage that only a low dose is needed for adjuvant activity (Oda et al., 2000; Rajput et al., 2007; Songa and $\mathrm{Hu} 2009$ ).

In a previous work, we prepared a combined inactivated EIV, EHV-1 and Tetanus toxoid (TT) vaccine adjuvanted with saponin and Alhydrogel, and evaluated the vaccine in guinea pigs. The obtained results were proved it to be safe, potent and stable at $4^{\circ} \mathrm{C}$ up to 12 months (Mohamed et al., 2017).
Thus, the present study was planned for monitoring the immune response of horses to combined inactivated EIV, EHV-1 and Tetanus toxoid (TT) vaccine adjuvanted with saponin and Alhydrogel.

\section{Materials and methods}

\subsection{Vaccine preparation}

The vaccine was prepared according to (Mohamed., et al 2018)

\subsection{Animals and vaccination}

Six adult apparently healthy horses with low antibody titer against EHV-1 and EIV, were used to evaluate the immunogenicity and potency of the vaccine.

Animals were divided to two groups; group A (four horses) were inoculated with two doses of combined inactivated EI, EHV-1 and TT vaccine adjuvanted with saponin and Alhydrogel one month apart. Each dose (4ml) was injected (I/M) and revaccinated after 6 months with the same dose and route. Group B (two horses) were kept at the same conditions as a control. All horses were observed carefully for recording any local or systemic reactions during the period of experiment.

\subsection{Sample collection}

Serum samples were collected from all horses at different intervals weekly in the first month, each two weeks after boostering and post revaccination, then monthly till 18 months post vaccination.

\subsection{Serological examination}

The immune response was evaluated using Haemagglutination inhibition (HI) test for EI virus after being treated with potassium periodate as described by (OIE, 2016), CFT and ELISA for EHV-1 (Crabb and Studdert, 1993; Sugiura et al., 1997; Singh et al., 2006) and $\mathrm{TN}$ test for toxin neutralizing antibodies after inactivation at $56^{\circ} \mathrm{C}$ for 30 minutes as previously described by (Barile et al., 1970).

\subsection{Statistics analysis:}


The obtained results were statistically analyzed using IBM SPSS statistics Version 21. Standard deviation values and $p$ values were calculated using anova test.

\section{RESULTS}

\subsection{Clinical signs}

All vaccinated and control horses were not showed any local or systemic reaction during the whole period of experiments by clinical examination.

\subsection{Immune response against EIV}

The antibody titer against EIV was evaluated using HI test. The developed antibody titer against EIV showed significant difference during the whole period of experiment $(\mathrm{P}=0.01)$, a mean titer was (72) at one-week post vaccination (WPV), which increased to (224) at 2 weeks then decrease again to (72) at $4 \mathrm{WPV}$.

The level of antibodies titer was increased again after booster dose at 4 weeks and reach to maximum at $3 \mathrm{MPV}$ (1152), then began to decline gradually till the time of revaccination (6 months).

After revaccination at $6 \mathrm{MPV}$, there was much higher HI antibody titer reached their maximum level (4608) at 8 and 9 MPV, then began to decline gradually till the end of the experiment as in table 1.

\section{3 immune responses against EHV-1}

The level of the developed antibodies against EHV-1 was determined using ELISA and reveled that no significant difference at time of sampling. After one WPV, the mean of ELISA titer was (430), reached to peak at 2WPV with mean (730), and decrease until 4WPV.
Following the booster dose at 4WPV, high level of antibodies was obtained and reached their maximum titer at $3 \mathrm{MPV}$ with mean ELISA titer (1490), then decrease until 6MPV (612.5). Furthermore, the ELISA antibody titer increased again and declined in the same manner after revaccination at $6 \mathrm{MPV}$ as shown in table 2.

In addition, the level EHV-1 of antibodies were determined also using CFT where it showed significant difference between level of all sampling time during experiment, it was (5) at one WPV and reached the peak at 3 WPV with mean CF titer (36), then decreased at 4WPV with mean CF titer (18). After the booster dose at the 4WPV, the level of antibodies was increased and reached to maximum titer at $2 \mathrm{MPV}$ with mean $\mathrm{CF}$ titer (72). At 6MPV, CF titer could not be detected in sera of horses.

The level of antibodies after revaccination at $6 \mathrm{MPV}$ was increased again and reached the peak at 7 MPV with mean CF titer (96), began to decrease gradually until $11 \mathrm{MPV}$ where $\mathrm{CF}$ antibodies could not be detected as in table 3.

\subsection{Immune responses to tetanus}

Regarding to immune response of vaccinated horses against tetanus toxoid, the $\mathrm{TN}$ antibodies level was $(1 \mathrm{IU} / \mathrm{ml})$ at one-week post vaccination. The level of antibodies reached their maximum titer $(6 \mathrm{IU} / \mathrm{ml})$ at 2 MPV, then the titer began to decline gradually till 6 months post vaccination after boostering dose at $4 \mathrm{WPV}$.

After revaccination at 6 months, Tetanus antitoxin titer increased to maximum level at $8 \mathrm{MPV}(16 \mathrm{IU} / \mathrm{ml})$ ), then decline gradually till 18 MPV with significant difference $(\mathrm{P}=0.01)$ as described in table 4. 
Table 1: EI -HI antibody titers in horses vaccinated with combined inactivated EI, EHV-1 \&TT vaccine

\begin{tabular}{|c|c|c|c|}
\hline \multirow{2}{*}{$\begin{array}{l}\text { Time } \\
\text { sampling }\end{array}$} & \multicolumn{3}{|c|}{ EI -HI antibody titers in sera of horses } \\
\hline & Mean/SD.T & $P$ value & Mean.C \\
\hline Prevaccination & $4 \pm 4.6$ & \multirow{24}{*}{0.01} & 8 \\
\hline$* * 1 \mathrm{WPV}$ & $72 \pm 40.2$ & & 0 \\
\hline $2 \mathrm{WPV}$ & $224 \pm 64$ & & 8 \\
\hline 3WPV & $176 \pm 69$ & & 8 \\
\hline$* * *$ (b) 4WPV & $72 \pm 40.26$ & & 0 \\
\hline $6 \mathrm{WPV}$ & $480 \pm 396.2$ & & 0 \\
\hline$* * * * 2 \mathrm{MPV}$ & $896 \pm 256$ & & 0 \\
\hline $3 \mathrm{MPV}$ & $1152 \pm 644.25$ & & 8 \\
\hline 4MPV & $896 \pm 256$ & & 0 \\
\hline $5 \mathrm{MPV}$ & $448 \pm 128$ & & 0 \\
\hline $\begin{array}{l}* * * * *(\quad \mathrm{R}) \quad 6 \\
\mathrm{MPV}\end{array}$ & $160 \pm 64$ & & 0 \\
\hline $6.5 \mathrm{MPV}$ & $1152 \pm 644.25$ & & 0 \\
\hline $7 \mathrm{MPV}$ & $2304 \pm 1288.5$ & & 0 \\
\hline $8 \mathrm{MPV}$ & $4608 \pm 2577.01$ & & 8 \\
\hline $9 \mathrm{MPV}$ & $4608 \pm 2577.01$ & & 8 \\
\hline $10 \mathrm{MPV}$ & $3840 \pm 3169.99$ & & 0 \\
\hline $11 \mathrm{MPV}$ & $2816 \pm 1536$ & & 0 \\
\hline $12 \mathrm{MPV}$ & $1792 \pm 512$ & & 8 \\
\hline 13MPV & $1664 \pm 768$ & & 0 \\
\hline 14MPV & $896 \pm 256$ & & 8 \\
\hline $15 \mathrm{MPV}$ & $704 \pm 384$ & & 0 \\
\hline $16 \mathrm{MPV}$ & $448 \pm 128$ & & 8 \\
\hline $17 \mathrm{MPV}$ & $352 \pm 192$ & & 0 \\
\hline $18 \mathrm{MPV}$ & $112 \pm 32$ & & 0 \\
\hline
\end{tabular}

**WPV: week post vaccination. $* * *(\mathbf{b})$ : booster dose $* * *$ MPV: month post vaccination $* * * * *(\mathbf{R})$ : revaccination. P-value significant (0.01-0.05) C:control group. T:Testgroup 
Field Evaluation of the prepared combined equine influenza, equine herpesvirus-1 and tetanus toxoid vaccine in horses

Table 2: EHV-1 antibody titers in horses vaccinated with combined inactivated EI, EHV-1 \&TT vaccine tested by ELISA

\begin{tabular}{|c|c|c|c|}
\hline \multirow{2}{*}{ Time of sampling } & \multicolumn{3}{|c|}{ EHV-1-ELI SA antibody titers in sera of horses } \\
\hline & Mean \pm SD & $\mathrm{P}$ value & Mean.C \\
\hline Prevaccination & $92.5 \pm 2.5$ & \multirow{21}{*}{0.01} & 95 \\
\hline$* * 1 \mathrm{WPV}$ & $430 \pm 43.96$ & & 95 \\
\hline $2 \mathrm{WPV}$ & $730 \pm 36.5$ & & 90 \\
\hline $3 \mathrm{WPV}$ & $590 \pm 43.96$ & & 90 \\
\hline $\begin{array}{l}* * * \\
\text { (b) } 4 \mathrm{WPV}\end{array}$ & $420 \pm 36.5$ & & 90 \\
\hline 6WPV & $1035 \pm 50.66$ & & 90 \\
\hline $\begin{array}{l}* * * * \\
2 \mathrm{MPV}\end{array}$ & $1227.5 \pm 64.48$ & & 90 \\
\hline $3 \mathrm{MPV}$ & $1490 \pm 82.22$ & & 95 \\
\hline 4MPV & $996.25 \pm 41.5$ & & 90 \\
\hline $5 \mathrm{MPV}$ & $812.5 \pm 26.29$ & & 90 \\
\hline$* * * * *(\mathrm{R}) 6 \mathrm{MPV}$ & $612.5 \pm 26.29$ & & 90 \\
\hline $6.5 \mathrm{MPV}$ & $1400 \pm 22.17$ & & 90 \\
\hline $7 \mathrm{MPV}$ & $1537.5 \pm 43.49$ & & 95 \\
\hline $8 \mathrm{MPV}$ & $2242.5 \pm 121.75$ & & 90 \\
\hline $9 \mathrm{MPV}$ & $1761.25 \pm 60.6$ & & 90 \\
\hline $10 \mathrm{MPV}$ & $1510 \pm 49.66$ & & 90 \\
\hline $11 \mathrm{MPV}$ & $987.5 \pm 85.04$ & & 100 \\
\hline $12 \mathrm{MPV}$ & $792.5 \pm 33.04$ & & 95 \\
\hline $13 \mathrm{MPV}$ & $642.5 \pm 33.04$ & & 90 \\
\hline $14 \mathrm{MPV}$ & $522.5 \pm 41.93$ & & 90 \\
\hline $15 \mathrm{MPV}$ & $377.5 \pm 12.58$ & & 90 \\
\hline $16 \mathrm{MPV}$ & $200 \pm 8.16$ & & 90 \\
\hline
\end{tabular}

**WPV: week post vaccination. $* * *(\mathbf{b})$ : booster dose $* * * * \mathbf{M P V}:$ month post vaccination $* * * * *(\mathbf{R})$ : revaccination.P-value significant $(0.01-0.05) \mathrm{C}$ :Control group T: Test group 
Table 3: EHV-1 antibody titer in horses vaccinated with combined inactivated EI, EHV-1 \&TT vaccine tested by CFT

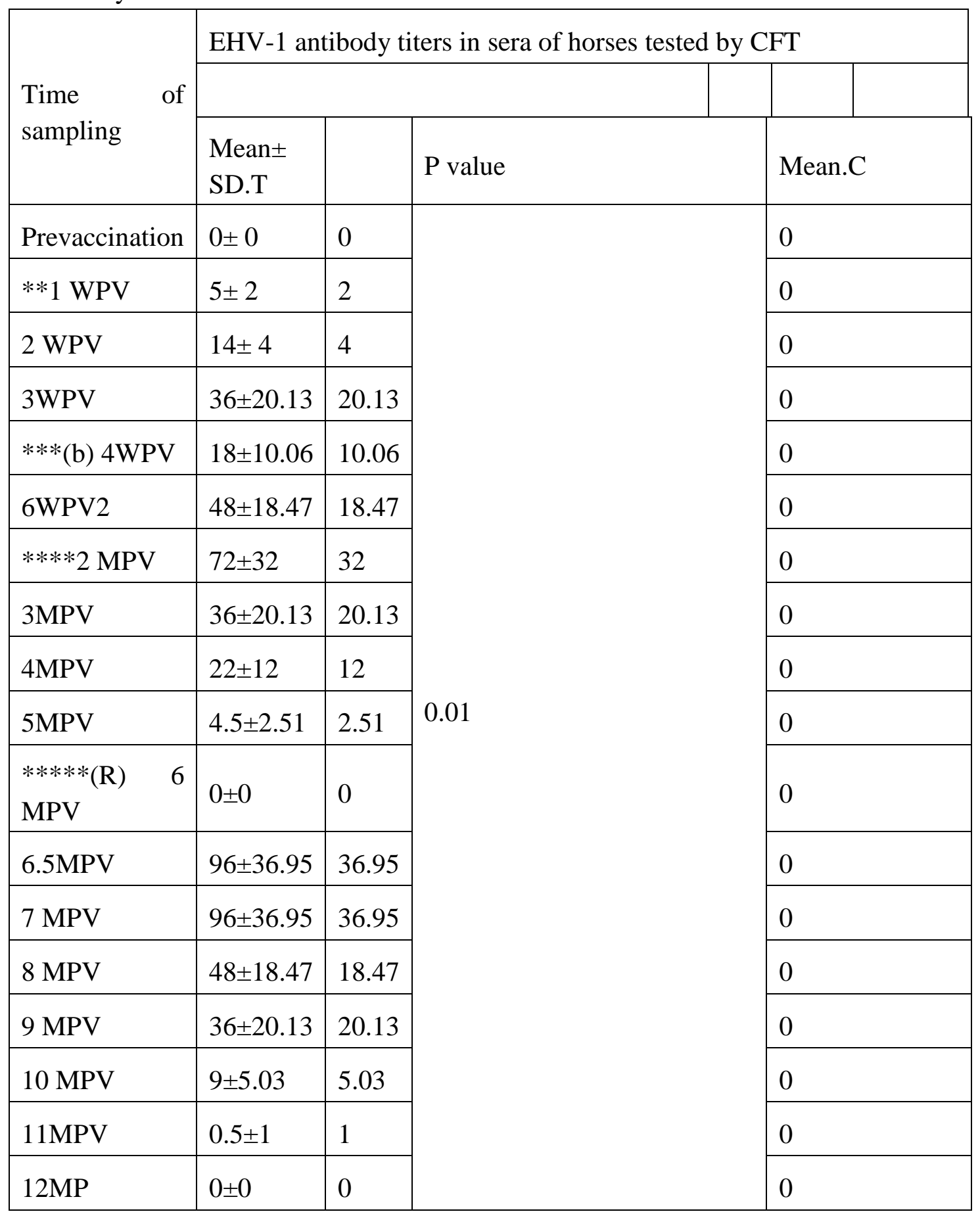

**WPV: week post vaccination. ***(b): booster dose ****MPV: month post vaccination $* * * * *(\mathbf{R})$ : revaccination. P-value significant $(0.01-0.05) \mathrm{C}$ : Control group T: Test group 
Field Evaluation of the prepared combined equine influenza, equine herpesvirus-1 and tetanus toxoid vaccine in horses

Table 4: Tetanus antitoxin titer in serum of horses vaccinated with combined inactivated EI, EHV-1 \&TT vaccine tested by toxin neutralization test.

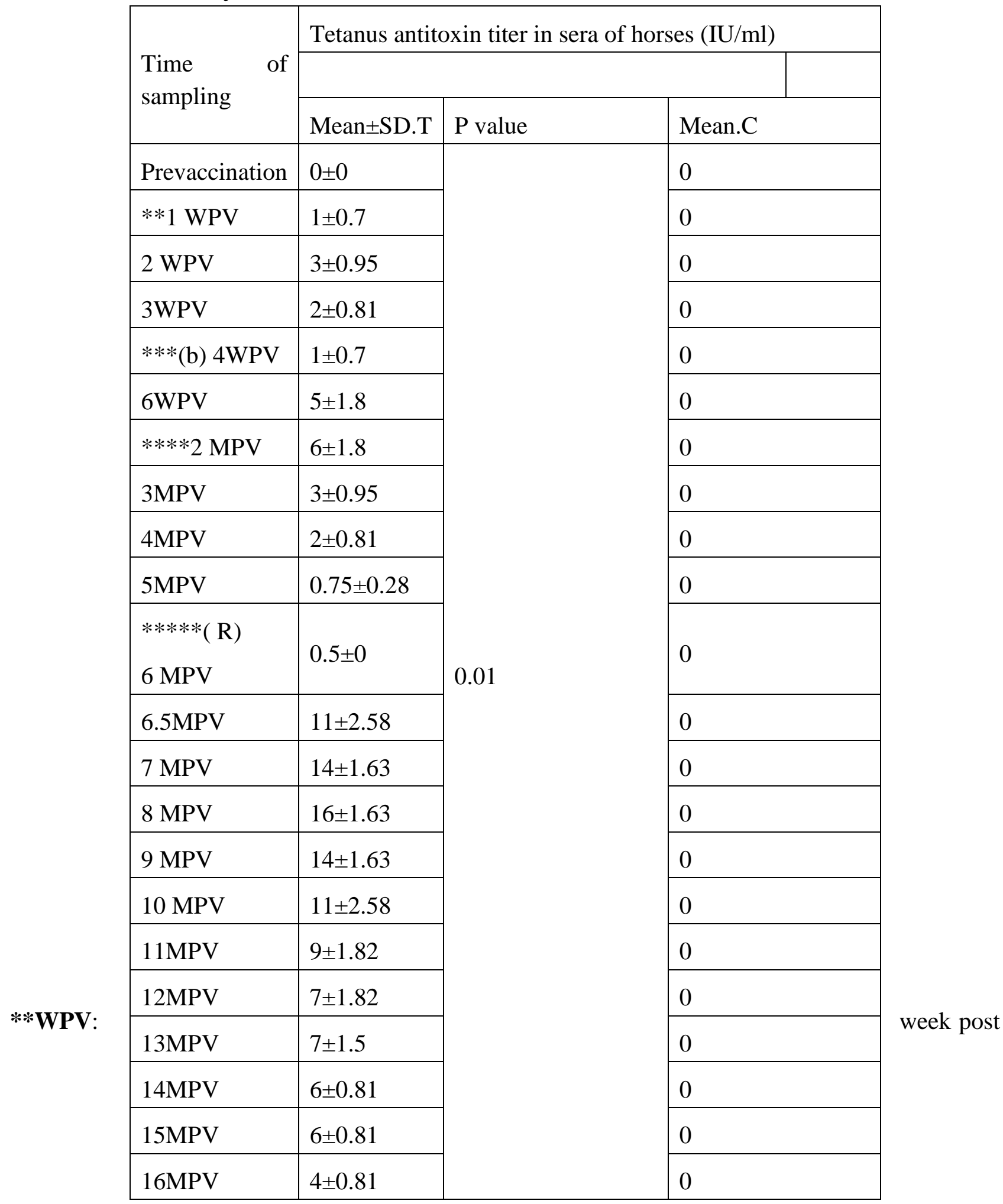

vaccination. $* * *(\mathbf{b})$ : booster dose $* * * * \mathbf{M P V}$ : month post vaccination $* * * * *(\mathbf{R})$ : revaccination. (IU): International antitoxin unit / $\mathrm{ml}$ of serum $\mathrm{P}$ Value significant (0.01- 0.05) C: Control group T: Test group 


\section{DISCUSSION}

Horses are commonly vaccinated to protect against pathogens which are responsible for diseases which are endemic within the general horse population, such as EIV and EHV-1, and against a variety of diseases which are less common but which lead to greater morbidity and mortality, such as tetanus (Holmes et al., 2006).

Measurement of immune response to vaccination can be a valuable indicator of vaccine efficacy, there is evidence for an association between serum antibody titers and protection from challenge infection in the case of EIV and tetanus (Liefman, 1981; Morley et al., 2000).

Moreover, the antibody responses to EHV-1 vaccination can be associated with clinical protection from respiratory disease and reduction of nasal viral shedding (Heldens et al., 2001; Kydd et al., 2006).

The combined inactivated EIV, EHV-1 and Tetanus toxoid (TT) vaccine adjuvanted with saponin and Alhydrogel was prepared and tested for safety according to (Mohamed et al., 2018). Immunogenicity of the vaccine was evaluated and monitored in horses up to 18 months post vaccination.

The absence of clinical signs of disease, or of any evidence of seroconversion to any antigen in the control group, provides strong evidence that all immune responses observed in this study were a result of vaccination.

EI-HI antibody titer started to appear after one-week post vaccination with mean titer (72). The highest titer of EI antibodies observed at $3 \mathrm{MPV}$ (1152) and $8 \& 9 \mathrm{MPV}$ (4608). and the protective level was persisted till the end of the experiment (18 months) as recommended by OIE, (2016) which mentioned that the antibody titer of each serum taken after the second vaccination and tested by HI test should not be less than 1/64 to be protective. The obtained results come in accordance with (Heldens et al., 2002), they found that the influenza components of the combination vaccine induced high antibody titers at two weeks after the second vaccination where after titers declined until the time of the third vaccination, then the titers rose rapidly again to remain high for at least 1 year.

The antibodies titer against EHV-1 using ELISA technique reached to maximum level after $3 \mathrm{MPV}$. These results agree with previous findings of (Bannai et. al., 2014; Rusli et. al., 2014) they found that ELISA antibodies begin to increase by day 14 post injection then reached its peak at 2 months.

At 14 days post booster dose and post revaccination there was significant increase in antibody titers 2-fold or more than the prebooster (4WPV) and pre-revaccination (6MPV) antibody titers as previously recommended by (OIE, 2017).

After the third vaccination, a seroresponse to EHV-1 was observed similar to that after the second vaccination. Eight months after the third vaccination, the average EHV-1 titer was low Similar o results were recorded by (Nashwa et al., 2016).

In the other hand, the level of antibodies in vaccinated horses against EHV1 was evaluated using CFT where the level of antibodies reached to highest level after 2 MPV and at 7 MPV after booster dose. The results come in accordance with (Singh et al., 2006; Paillot et al., 2008) who reported that the humeral response stimulated by vaccination is generally quantified by the titration of CF antibodies for EHV-1; seroconversion is defined as a significant increase (2-fold) of antibody titers. 
The positive protective $\mathrm{CF}$ antibody titers $\geq 20$ were detected from 6WPV to 4MPV and after revaccination from 6.5 to 9MPV. These results come parallel to those obtained by (Burrows et al., 1984) who stated that horses with $\mathrm{CF}$ antibody titer $\geq 20$ showed reduction in the incidence and duration of clinical signs after challenge

Tetanus Antitoxin titers remain within the protective level up to 18 months $(0.01$ $\mathrm{IU} / \mathrm{ml}$ ) as recommended by (Löhrer et al., 1970; Heldens et al., 2001) who stated that a Serum neutralization antibody titer in mice have been demonstrated to correlate very well with protection against tetanus in horses. Antibody titers of $0.01 \mathrm{IU} / \mathrm{m} 1$ were shown to be protective.

However, recent equine vaccines trials have indicated that the efficacy of vaccines are unaffected by their administration as monovalent or polyvalent formulations (Heldens et al., 2002).

\section{CONCLUSION}

The vaccinated horses with combined inactivated EIV, EHV-1 and Tetanus toxoid (TT) vaccine adjuvanted with saponin and Alhydrogel were developed high antibody titers, above the level for clinical protection against influenza and tetanus until 12 months and 6 months for EHV-1 after the third vaccination.

\section{REFERENCES}

Barile, M.F., Hardegree, M.C. and Pittman, M. 1970. Immunization against neonatal tetanus in New Guinea. 3 The toxin-neutralization test and the response of guinea pigs to the toxoids as used in the immunization schedules in New Guinea. Bull. WHO, 43, 453459.

Bannai, H., Mae, N., Ode, H., Nemoto, M., Tsujimura, K., Yamanaka, T., Kondo,
T. and Matsumura, T. 2014. Successful control of winter pyrexias caused by EHV-1 in Japanese training centers by achieving high vaccination coverage. Clin. Vaccine Immunology., 21, 10701076.

Burrows, R., Goodridge, D. And Denyer, M.S. 1984. Trials of an inactivated equid herpesvirus-1 vaccine: Challenge with a subtype 1 virus. Vet. Rec., 14, 369- 374.

Bryant, N.A., Rash, A.S., Woodward, A.L., Medcalf, E., Helwegen, M., Wohlfender, F., Cruz, F., Herrmann, C., Borchers, K. And Tiwari, A. 2010.' Isolation and characterization of equine influenza viruses (H3N8) from Europe and North America from 2008 to 2009. Vet. Microbiol, 147, 19-27.

Crabb, B.S. and Studdert, M.J. 1993. Epitopes of glycoprotein $\mathrm{G}$ of equine herpesviruses 4 and 1 located near the C termini elicit type-specific antibody responses in the natural host. J. Virol., 67, 6332-6338.

Heldens, J., Kersten, A., Weststrate, M. and Van Den Hoven, R. 2001. Vaccinology: Duration of immunity induced by an adjuvanted and inactivated equine influenza, tetanus and equine herpesvirus 1 and 4 combination vaccine. Vet. Q., 23, 210217.

Heldens, J.G., Van De Wouw, J.C.A. and Van Loon, A.A.W.M. 2002. An updated equine influenza vaccine and an equine influenza herpesvirus combination vaccine containing an Immunostimulant adjuvant provoke equal antibody levels in young foals throughout the primary vaccination course. Vet. J. 164, 288-291. 
Holmes M A, Hugh G.G., Andrea K. K., Steve H., Cormac B., Craig B., Robert H.E. and Lunn D.P. 2006. Immune responses to commercial equine vaccines against equine herpesvirus-1, equine influenza virus, eastern equine encephalomyelitis, and tetanus. Vet. Immunol. Immunopathol., 111, 67-80.

Inić-Kanada, A.B., Stojanović, M.M., Živković, I.P., Petrušić, V.Ž. and Dimitrijević, L.A. 2009. The monoclonal antibody 26 raised against tetanus toxoid also recognizes tetanus toxin and $\beta 2$-glycoprotein I-its binding properties in vitro and potential applications. Journal of the Serbian chemical society, 74, 245-257.

Jansen, B.C. and Knoetze, P.C. 1979. The immune response of horses to tetanus toxoid. Onderstepoort J. Vet. Res. 46, 211-216.

Kydd, J., Slater, J., Osterrieder, N., Lunn, D., Antczak, D., Azab, W., Balasuriya, U., Barnett, C., Brosnahan, M. and Cook, C. 2006. Third international havemeyer workshop on equine herpesvirus type 1. Equine Vet. J., 44, 513-517.

Lindblad, E.B. 2004. Aluminium adjuvantsin retrospect and prospect. Vaccine, 22, 3658-3668.

Liefman, C.E. 1981. Active immunisation of horses against tetanus including the booster dose and its application. Aust. Vet. J., 57, 57-60.

Löhrer, J. And Radvila, P. 1970. Active tetanus prophylaxis in the horse and the duration of immunity. Schweiz. Arch. Tierheilkd., 112, 307-314.

Lunn, D.P. And Townsend, H.G.G., 2000. Equine vaccination. Vet. Clin. North Am. Equine Pract. 16, 199-226.
Mesquita A L P., Andressa Ferrari Arévaloa, Dennis A. Zanattoa, Samantha Ive Miyashiroa, Elenice Maria Sequetin Cunhab, Maria Do Carmo Custódio De Souzab, Eliana Monteforte Cassaro Villalobosb, Cláudia Madalena Cabrera Moria, Paulo César Maiorkaa, Enio Moria, C. 2017. Equine herpesvirus type 1 induces both neurological and respiratory disease in Syrian hamsters. Vet. Microbiol., 203,117-124.

Mohamed, B., Abdelmoneim. M., Nashwa. K.M., Selim, A., Wafaa A. 2017. Trial for Preparation of Combined Equine Influenza, Equine Herpesvirus-1 and Tetanus Toxoid Vaccine. Benha Vet. Med. J.(34)1

Morley, P.S., Townsend, H.G.G., Bodgan, J.R. And Haines, D.M. 2000. Risk factors for disease associated with influenza virus infections during three epidemics in horses. J. Am. Vet. Med. Assoc.216, 545-550.

Nashwa, K.M., Eman, M.E., Nehal S.S., Fatma, F.Y., El-Kabbany, M.M.A. And Soliman I.M.A. 2016. Duration of immunity induced by combined vaccine against Equine influenza and Equine herpesvirus-1. VMJG, 62, 1110-1423.

Oie 2016. Equine Influenza. Chapter 2.5.7, 115

Oie 2017. Equine rhinopneumonitis Chapter 2.5.9, 1-13.

Oda, K., Matsuda, H., Murakami, T., Katayama, S., Ohgitani, T. And Yoshikawa M. 2000. Adjuvant and hemolytic activity of 74 saponins derived from medicinal and food plants. Biol. Chem., 381, 67-74. 
Paillot, R., Case, R., Ross, J., Newton, R. \& Nugent, J. 2008a. Equine herpes virus1: virus, immunity and vaccines. Open Vet. Sci. J., 2, 68-91.

Rajput, Z.I., Hu, S.H., Xiao, C.W. And Arijo, A.G. 2007. Adjuvant effects of saponins on animal immune responses. J. Zhejiang Univ. Sci. B. 8, 153-161

Rusli, N. D., Mat, K. B. \& Harun, H. C. 2014. A review: interactions of equine herpesvirus-1 with immune system and equine lymphocyte. Open J Vet. Med., 4, 294 .
Singh, B., Tandon, S. And Virmani, N. 2006. Immune responses to inactivated oil adjuvanted Equine Herpes Virus-1 using different emulsifiers in horses. Indian J Biotech. 5, 42-46.

Sugiura, T., Kondo, T., Matsumura, T., Imagawa, H., Kamada, M., Songa X. And Hua S., 2009. Adjuvant activities of saponins from traditional Chinese medicinal herbs. Vaccine, 27, 48834890. 